\title{
Long Arithmetic Progressions of Primes: Some Old, Some New
}

\author{
By Paul A. Pritchard
}

\begin{abstract}
The results are reported of an extensive search with a computer for "long" arithmetic progressions of primes. Such progressions with minimum last term are now known for all lengths up to and including nineteen.
\end{abstract}

A long-standing conjecture of Hardy and Littlewood [5] - the "prime $k$-tuples conjecture" - is that if $a_{1}, \ldots, a_{k}$ do not form a complete residue system for any prime $p$, then there are infinitely many values of $x$ such that $x+a_{1}, \ldots, x+a_{k}$ are all prime. The conjecture is actually given in [5] in a much stronger form, viz., an asymptotic formula for the number of such values of $x$ not exceeding a given bound. Two interesting implications of the prime $k$-tuples conjecture are that there are infinitely many twin primes, and that for all $n>0$ there are infinitely many arithmetic progressions of $n$ primes (denoted PAPs in the sequel). We are concerned herein with the second of these.

Very little about our subject has been proved. Roughly speaking, the present state of knowledge is that $n$ can be $3 \frac{1}{2}$. More precisely, Chowla [1] showed that there are infinitely many PAPs of length 3, and, more recently, Grosswald [2] established the validity of the Hardy-Littlewood asymptotic formula in that case, and Heath-Brown [6] has shown that there are infinitely many arithmetic progressions consisting of three primes and an "almost prime" (a number with at most two prime factors).

With the aid of computers, PAPs have been discovered that are substantially longer than those guaranteed to exist by these or other available theorems. Before our work, the longest known PAP had length 17 [13]. In [11] we announced a PAP of length 18 discovered by a search that was then still in progress, and undertook to report again on completion of the search. This paper contains the promised report and also the results of other, shorter computations that fill in some gaps left in earlier work. The highlight is a PAP of length 19. Note that we do not consider PAPs with so-called "negative primes", unlike, e.g., [8].

The new results reported herein were discovered by programs whose design is presented in detail in [10]. Our major computation was a search for all PAPs, of

Received May 9, 1984; revised October 22, 1984.

1980 Mathematics Subject Classification. Primary 10-04; Secondary 10L20. 
length at least 17 , that have a common difference divisible by 9699690 - the product of the primes $\leqslant 19-$ and no term exceeding $30001 \times 9699690$. (A PAP of length 19 must have a common difference of this form or else have first term 19; the latter case is unlikely and was checked separately.) Our search ran in the background on two Digital Equipment Corporation VAX-11/780s in the Department of Computer Science at Cornell University, from 6 October 1982 to 18 March 1984, and consumed almost 14000 hours of computer time.

Table 1 is an update of Table 2 of [3]. It lists for each $m, 1<m \leqslant 19$, the PAP of length $m$ with minimum last term. For the heuristic argument leading to the estimates in the last column, see [3]. The PAPs for $m=14,15,18,19$ have been discovered since [3] appeared. Also new is the knowledge that each PAP listed is indeed the one with minimum last term; previously, this was known only for $m \leqslant 10$.

\section{TABLE 1}

The known PAPs with minimum last term

\begin{tabular}{|r|l|r|r|}
\hline$m$ & $\begin{array}{c}\text { PAP of length } m \text { with minimal last term } \\
(k=0,1,2, \ldots, m-1)\end{array}$ & $\begin{array}{r}\text { estimated } \\
\text { last term }\end{array}$ \\
\hline 2 & 2,3 & 3 & 2 \\
3 & $3,5,7$ & 7 & 2 \\
4 & $5,11,17,23$ & 23 & 2 \\
5 & $5,11,17,23,29$ & 29 & 29 \\
6 & $7+30 k$ & 157 & 92 \\
7 & $7+150 k$ & 907 & 497 \\
8 & $199+210 k$ & 1669 & 1406 \\
9 & $199+210 k$ & 1879 & 5086 \\
10 & $199+210 k$ & 2089 & 24310 \\
11 & $110437+13860 k$ & 249037 & 177300 \\
12 & $110437+13860 k$ & 262897 & 829800 \\
13 & $4943+60060 k$ & 725663 & 5582000 \\
14 & $31385539+420420 k$ & 36850999 & $2.332 \times 10^{7}$ \\
15 & $115453391+4144140 k$ & 173471351 & $1.137 \times 10^{8}$ \\
16 & $53297929+969960 k$ & 198793279 & $6.793 \times 10^{8}$ \\
17 & $3430751869+87297210 k$ & 4827507229 & $5.774 \times 10^{9}$ \\
18 & $4808316343+717777060 k$ & 17010526363 & $3.303 \times 10^{10}$ \\
19 & $8297644387+4180566390 k$ & 83547839407 & $2.564 \times 10^{11}$ \\
20 & $?$ & $?$ & $1.261 \times 10^{12}$ \\
\hline
\end{tabular}

Sierpinski defines $g(x)$ to be the maximum number of terms in an arithmetic progression of primes not greater than $x$. The least $x, l(x)$, for which $g(x)$ takes the values $0,1, \ldots, 19$ can be read off from Table 1 , thereby correcting and extending the information in [4].

Table 2 is an adaption and extension of Table 1 on p. 11 of [4]. It gives, for each $n, 12 \leqslant n \leqslant 19$, the first-discovered PAP with length $n$ and the PAP of length $n$ with smallest last term. Note that the first-discovered PAPs of lengths 13, 17, and 19 are also those with smallest last term, and that the first-discovered PAPs of lengths 14 and 15 are initial parts of Root's PAP of length 16. 
TABLE 2

Some PAPs and their discoverers

\begin{tabular}{|l|r|r|r|l|}
\hline$n$ & $\begin{array}{r}\text { common } \\
\text { difference }\end{array}$ & first term & last term & \multicolumn{1}{|c|}{ discovery } \\
\hline 12 & 30030 & 23143 & 353473 & V. A. Golubev, 1958 (see [8]) \\
12 & 13860 & 110437 & 262897 & E. Karst, 1967 ([8]) \\
13 & 60060 & 4943 & 725663 & V. N. Seredinskij, 1963 (see [8]) \\
14 & 223092870 & 2236133941 & 5136341251 & S. C. Root, 1969(see [7]) \\
14 & 420420 & 31385539 & 36850999 & P. A. Pritchard, 1983 \\
15 & 223092870 & 2236133941 & 5359434121 & S. C. Root, 1969 (see [7]) \\
15 & 4144140 & 115453391 & 173471351 & P. A. Pritchard, 1983 \\
16 & 223092870 & 2236133941 & 5582526991 & S. C. Root, 1969(see [7]) \\
16 & 9699690 & 53297929 & 198793279 & S. Weintraub, 1976 ([12]) \\
17 & 87297210 & 3430751869 & 4827507229 & S. Weintraub, 1977 ([13]) \\
18 & 9922782870 & 107928278317 & 276615587107 & P. A. Pritchard, 1982 ([11]) \\
18 & 717777060 & 4808316343 & 17010526363 & P. A. Pritchard, 1983 \\
19 & 4180566390 & 8297644387 & 83547839407 & P. A. Pritchard, 1984 \\
\hline
\end{tabular}

We know of no PAP of length 20 (or greater). The known PAPs of length at least 18 are given in Table 3. (The two PAPs of length 18 that can be obtained from the one of length 19 are not listed.)

TABLE 3

The known PAPs of length at least 18

\begin{tabular}{|c|r|r|r|}
\hline length & \multicolumn{1}{|c|}{ first term } & \multicolumn{1}{c|}{$\begin{array}{c}\text { common } \\
\text { difference }\end{array}$} & \multicolumn{1}{c|}{ last term } \\
\hline 18 & 4808316343 & 717777060 & 17010526363 \\
19 & 8297644387 & 4180566390 & 83547839407 \\
18 & 64158606367 & 2735312580 & 110658920227 \\
18 & 2518035911 & 7536659130 & 130641241121 \\
18 & 115936060313 & 3103900800 & 168702373913 \\
18 & 98488875263 & 5169934770 & 186377766353 \\
18 & 170263333103 & 5063238180 & 256338382163 \\
18 & 107928278317 & 9922782870 & 276615587107 \\
18 & 51565746467 & 13889956080 & 287694999827 \\
\hline
\end{tabular}

Grosswald [2] showed that with an additional assumption, the Hardy-Littlewood asymptotic estimate of the number of PAPs of length $n$ with no term exceeding $x$ could be cast in an easily computable form. Grosswald and Hagis [3] showed that, for relatively short progressions, the estimate is reasonably accurate. Our major calculation was designed to test Grosswald's estimate, using much longer PAPs than had hitherto been employed.

Before presenting the results of this test, it is necessary to adapt Grosswald's estimate so that it applies to PAPs with a restricted form of common difference. Fortunately, an estimate is implicit in Grosswald's argument. Let us define $N_{m, n}(x)$ 
to be the number of PAPs of length $m$ with all terms $\leqslant x$ and common difference a multiple of the product of the primes $\leqslant n$. We have

$$
N_{m, n}(x) \sim \prod_{m<p \leqslant n} \frac{1}{p+1-m} \cdot \frac{c_{m} x^{2}}{\log ^{m} x}
$$

where

$$
c_{m}=\frac{1}{2(m-1)} \prod_{p>m}\left(\left(\frac{p}{p-1}\right)^{m-1} \frac{p-(m-1)}{p}\right) \prod_{p \leqslant m}\left(\frac{1}{p}\left(\frac{p}{p-1}\right)^{m-1}\right) .
$$

The right-hand side of (1) is actually the dominant term of an infinite series for $N_{m, n}(x)$. Grosswald [2] gives a computable expression for the next term, which contributes significantly for the numbers under consideration, and which was therefore incorporated into our calculations. The third term was not computed, mainly because the calculations are very involved, but also because we did not expect it to significantly alter our estimates.

Table 4 shows the actual versus estimated counts of $N_{m, n}(x)$ for our experiment, in which $x=300001 \times 9699690, n=19$, and $m=17,18,19$. As reported in [3], and as is reasonable for an asymptotic estimate, the predicted value is most accurate when it is largest. We assure the skeptical reader that the successive powers of 10 in the second column are the exact counts of PAPs found.

TABLE 4

Actual versus predicted numbers of PAPs

\begin{tabular}{|c|c|c|}
\hline \multirow{2}{*}{ length } & \multicolumn{2}{|c|}{ Number of PAPs } \\
\cline { 2 - 3 } & actual & predicted \\
\hline 17 & 100 & 85.7 \\
18 & 10 & 13.1 \\
19 & 1 & 1.9 \\
\hline
\end{tabular}

Acknowledgments. The truly enormous computation reported above was made possible by the assistance I received from the staff who maintained the computer systems in the Department of Computer Science at Cornell University. My special thanks go to Dean Krafft, for his supportive administrative decisions, and Mike Hammond, for his technical assistance.

Department of Computer Sciences

Purdue University

West Lafayette, Indiana 47907

1. S. Chowla, "There exist an infinity of 3-combinations of primes in A. P.," Proc. Lahore Philos. Soc., v. 6, 1944, pp. 15-16.

2. E. Grosswald, “Arithmetic progressions that consist only of primes," J. Number Theory, v. 14, 1982, pp. 9-31.

3. E. Grosswald \& P. Hagis, JR., "Arithmetic progressions consisting only of primes," Math. Comp., v. 33, 1979, pp. 1343-1352.

4. R. K. GUY, Unsolved Problems in Number Theory, Springer-Verlag, New York, 1981.

5. G. H. HARDY \& J. E. LitTLEwOOD, "Some problems of 'partitio numerorum' III: on the expression of a number as a sum of primes," Acta Math., v. 44, 1923, pp. 1-70. 
6. D. R. Heath-Brown, “Three primes and an almost prime in arithmetic progression," J. London Math. Soc. (2), v. 23, 1981, pp. 396-414.

7. E. KARST, "12-16 primes in arithmetical progression," J. Recreational Math., v. 2, 1969, pp. 214-215.

8. E. KARST, “Lists of ten or more primes in arithmetical progressions," Scripta Math., v. 28, 1970, pp. 313-317.

9. E. KARST \& S. C. Root, “Teilfolgen von Primzahlen in arithmetischer Progression," Anz. Österreich. Akad. Wiss. Math.-Natur. Kl., 1972, pp. 19-20 (see also pp. 178-179).

10. P. A. Pritchard, "A case study of number-theoretic computation: searching for primes in arithmetic progression," Sci. Comput. Programming, v. 3, 1983, pp. 37-63.

11. P. A. Pritchard, "Eighteen primes in arithmetic progression," Math. Comp., v. 41, 1983, p. 697.

12. S. WeintraUB, "Primes in arithmetic progression," BIT, v. 17, 1977, pp. 239-243.

13. S. Weintraub, “Seventeen primes in arithmetic progression," Math. Comp., v. 31, 1977, p. 1030. 